\title{
1 SmartRNASeqCaller: improving germline variant calling from RNAseq
}

2 Mattia Bosio ${ }^{1,2}$, Alfonso Valencia ${ }^{1,2,3}$, Salvador Capella-Gutierrez ${ }^{1,2, *}$

\section{3}

4 1: Barcelona Supercomputing Center (BSC), C/Jordi Girona 29, 08034, Barcelona, Spain

5 3: Spanish National Bioinformatics Institute (INB), ELIXIR-ES.

6 3: ICREA, Pg. Lluís Companys 23, 08010, Barcelona, Spain

7 * Corresponding author, mail : salvador.capella@bsc.es

\section{Abstract}

\section{Background:}

11 Transcriptomics data, often referred as RNA-Seq, are increasingly being adopted in 12 clinical practice due to the opportunity to answer several questions with the same data -

13 e.g. gene expression, splicing, allele-specific expression even without matching DNA.

14 Indeed, recent studies showed how RNA-Seq can contribute to decipher the impact of 15 germline variants. These efforts allowed to dramatically improved the diagnostic yield in

16 specific rare disease patient cohorts. Nevertheless, RNA-Seq is not routinely adopted for 17 germline variant calling in the clinic. This is mostly due to a combination of technical noise 18 and biological processes that affect the reliability of results, and are difficult to reduce 19 using standard filtering strategies.

\section{Results:}

22 To provide reliable germline variant calling from RNA-Seq for clinical use, such as for 23 mendelian diseases diagnosis,, we developed SmartRNASeqCaller: a Machine Learning 24 system focused to reduce the burden of false positive calls from RNA-Seq. Thanks to the 25 availability of large amount of high quality data, we could comprehensively train 26 SmartRNASeqCaller using a suitable features set to characterize each potential variant. 
27 The model integrates information from multiple sources, capturing variant-specific

28 characteristics, contextual information, and external sources of annotation. We tested our

29 tool against state-of-the-art workflows on a set of 376 independent validation samples

30 from GIAB, Neuromics, and GTEx consortia. SmartRNASeqCaller remarkably increases

31 precision of RNA-Seq germline variant calls, reducing the false positive burden by $50 \%$

32 without strong impact on sensitivity. This translates to an average precision increase of

$3320.9 \%$, showing a consistent effect on samples from different origins and characteristics.

\section{Conclusions:}

36 SmartRNASeqCaller shows that a general strategy adopted in different areas of applied

37 machine learning can be exploited to improve variant calling. Switching from a naïve

38 hard-filtering schema to a more powerful, data-driven solution enabled a qualitative and

39 quantitative improvement in terms of precision/recall performances. This is key for the

40 intended use of SmartRNASeqCaller within clinical settings to identify disease-causing

41 variants.

\section{Keywords:}

43 RNA-Sequencing, variant calling, machine learning, transcriptomics

\section{Background}

46 Being able to associate genomic variation to phenotypic traits is a long-lasting question

47 and fundamental task for omics data analysis. Massive adoption of next sequencing

48 technologies enabled the discovery of causal links between genetic variants and

49 phenotypes. This is especially true for monogenic mendelian diseases $(1,2)$ and in most

50 of cancer studies (3-5). On one side, NGS data have been used to elucidate the genetic 
51 origin of many diseases, with successful diagnoses in $41 \%$ of cases overall. On the other

52 side, hundreds of cancer driver genes, and thousands of putative cancer-driver mutations

53 have been identified using NGS with important consequences for diagnosis and

54 treatment.

55 Whole-genome sequencing (WGS) and whole-exome sequencing (WES) are commonly

56 adopted both in multicenter studies with thousands of patients (6-8), and increasingly in

57 clinical daily practice (2,9-11). In parallel, initiatives like GTEx (8) showed how RNA-Seq

58 data enriched the picture of genome-phenome relationships, for example defining tissue-

59 specific expression and eQTLs. The potential to answer multiple questions

60 simultaneously from RNA-Seq e.g. gene expression, splicing detection, allele specific

61 expression (12-15), jointly with its reduced costs, convinced an increasingly large share

62 of scientists to adopt RNA-Seq in their analyses.

63 Using RNA-Seq to call germline variants can be beneficial in clinical settings, for example

64 for Mendelian and common diseases studies. While RNA-Seq does not require additional

65 laboratory experiments if data are already collected, it can enhance the information from

66 samples without matching DNA $(16,17)$. Indeed, it has been shown to significantly

67 improve the diagnostic yield for Rare Diseases (18) when used jointly with DNA data, and

68 thoroughly processed by field-experts. These recent results show an opportunity to

69 develop tools to automatically enhance the information that can be extracted from an ever

70 growing number of RNA-Seq samples. Such tools need to deal with a whole set of

71 technical challenges i.e. split read mapping, alternative splicing, RNA-Edit, RNA

72 polymerase errors during transcription, and allele specific expression $(12,15,16)$ hindering

73 the reliability of RNA-Seq variant calls. A fundamental step for a broader RNA-Seq

74 adoption in clinical settings for variant discovery and prioritization is to reduce the burden

75 of false positive calls. A number of workflows have been developed to reliably call and

76 filter germline variants from RNA-Seq including SNPiR, Opossum or eSNV-detect 
$77(16,19,20)$. Those workflows rely on a set of hard-filtering rules implying a trade-off

78 between quality and quantity of called variants. Such filtering schemas have a limited

79 ability to capture complex patterns, and to discriminate true germline calls from the rest.

80 In this work, we developed SmartRNASeqCaller, a machine-learning module to

81 accurately predict germline variants from RNA-Seq. It makes use of a Random Forest

82 (RF) model that integrates intrinsic variant features with external annotations.

83 SmartRNASeqCaller then generates a data-driven nonlinear predictor for germline

84 variants, harnessing the power to detect complex feature relationship from a massive

85 high-quality training dataset. With SmartRNASeqCaller we aim to improve existing state-

86 of-the-art in discriminating true germline variants from the rest by adopting a more

87 powerful and integrative approach than the hard-filtering strategy used in most of the

88 existing workflows. The overall objective is to minimize the burden of false positive calls

89 from RNA-Seq to call variants with comparable reliability to WGS/WES results. Similar to

90 other biomedical research fields where machine learning techniques are used $(21,22)$, the

91 main novelty of our approach relies on learning complex patterns to discriminate if a given

92 call is a true germline variant.

93 SmartRNASeqCaller can be applied as a standalone module to refine the results from

94 previous variant calling workflows without requiring a full sample re-analysis. In this work,

95 we provide SmarRNASeqCaller as a plugin to the GATK best-practices workflow. This

96 module can be easily integrated into any variant calling workflow, as long as it provides

97 an aligned BAM file, and a VCF file with the variants to be classified.

98 In order to compare the performance of this newly proposed module, we benchmarked

99 the impact of including SmartRNASeqCaller as an additional step after using the GATK

100 best practices workflow against only using the GATK workflow and against SNPiR. We

101 analysed a set of 10 independent high-quality samples from Neuromics consortium (23),

102 as well as on GIAB sample NA12878 (24). We then compared SmartRNASeqCaller 
103 impact when applied to the resulting variants from the GATK best practices pipeline on

104365 samples from GTEx consortium, collected from 5 tissues from 73 donors. These

105 independent tests serve to confirm the utility of the method in improving germline variant

106 call precision for clinical applications through specific real use-cases.

\section{Implementation}

108 We have implemented an effective tool to post-process variant calling results from RNA-

109 Seq to reliably identify germline variants. This tool is designed to be used as an additional

110 step in conventional variant calling workflows. It integrates ideas and resources from the

111 literature $(12,13,19,25)$ within a machine learning framework. The driving approach is to

112 use Random Forest (RF), a machine learning technique, to generate a model that is able

113 to discriminate true germline variants from the rest. This process is possible by identifying

114 complex patterns based on variants annotated features coming from multiple sources.

115 SmartRNASeqCaller is divided in two main steps. First, each variant is annotated with a

116 set of 20 features (table 1). Seven out of them are intrinsic properties including variant

117 type and length, as well as contextual features including external annotations such as the

118 variant in a RepMask region from the UCSC annotation (26), and whether it is annotated

119 into a RNA-Edit site from $(25,27)$. In parallel, the caller specific features include GATK

120 specific quality values, as well as others such as BaseQRankSum, MQRankSum and

121 ClippingScore. Second, each variant is processed by a classifier that estimates the

122 likelihood of being a true germline variant e.g. appearing in the genomic DNA.

123 Importantly, this classifier model has been generated using a RF approximation, trained

124 on a set of high-quality matched samples of WGS and RNA-Seq with more than 600'000

125 variants. 
126

127

128

129

130

131

146 applied.

\section{Baseline variant calling workflow}

148 Prior to the application of SmartRNASeqCaller, we processed RNA-Seq from GIAB and 149 Neuromics with GATK RNA-Seq best practices workflow, available at this repository 150 [https://github.com/inab/RDConnect RNASeq]. This workflow produces two files i) an 
151 aligned BAM file, which is obtained with the STAR v2.35a aligner and uses GATK 3.6.0

152 for subsequent processing steps (24), and ii) a VCF file with the initial set of candidate

153 variants that will be used as input for SmartRNASeqCaller.

154 GTEx samples were already aligned with TopHat 1.4, thus we used the provided BAM file

155 as input for the variant calling workflow. This difference in the original alignment step

156 represents an opportunity to evaluate the SmartRNASeqCaller performance on data

157 generated following an alternative approach to the one used to train this classifier.

\section{SmartRNASeqCaller training}

159 We used 665,178 called variants from 10 matched DNA and RNA-Seq samples from the

160 Neuromics Consortium as our training set. The training dataset size allows to build a

161 model for discriminating true germline variants from the rest using a Random Forest (RF)

162 algorithm with sufficient data to reduce potential overfitting to the training set. We chose

163 to use a RF-based algorithm considering the available number of variants in the training

164 set and the need to detect complex patterns without a predefined structure. Other

165 methods like deep learning require at least a spatial data-structure for building a model.

166 Moreover, RF automatically deals with different data types e.g. binaries, qualitative and

167 quantitative, without requiring prior normalization step, and it is robust to class

168 imbalancing (27,38). Conversely, Support Vector Machine (SVM) and others classical

169 regression models tend to be more sensitive to the classes unbalanced and, in addition,

170 their performances depend on data normalization strategies (38). Finally, a key aspect for

171 choosing RF over other potential options is the robustness of this approximation to over-

172 fitting since we want the model to have consistent performances on novel samples.

173 An initial set of 20 features, generic and GATK specific, were analyzed for training the

174 model (table 1). We employed a recursive feature elimination strategy with 10 fold cross

175 validation applied on the training variants set (as shown in Figure 1A) to select the best 
176 feature set for classification. Analysing the results in Figure 1A, we chose 11 features,

177 given that the overall trade-off among average accuracy, accuracy variance, and

178 overfitting potential of the model. With only 11 features, the overall model accuracy is

179 close to the maximum, is quite compact, and is able to generate robust predictions.

180 Importantly, all excluded features fall very close to some selected feature in the tSNE plot

181 in Figure 1B, suggesting that the information content from the excluded features are

182 already provided by other features in the model. The model features, together with the

183 excluded ones are listed in Table 1. We used the R (version 3.5.1) modules RangeR and

184 caret for the model training and evaluation.

185 The selected 11 features are a collection of heterogeneous variant descriptions (Figure

186 1B and Table 1). It includes intrinsic variant properties as well contextual ones including

187 GATK specific features, the later give an assessment of the trustworthiness of the variant

188 call (table 1). We also included variants annotation from external datasets and genomic

189 context e.g. variant overlapping with an homopolymeric stretch of $5 \mathrm{bp}$ or more, variant

190 overlapping with the 4bp intronic region of exon-intron junctions, variant annotated as

191 RNA-Edit events from (16,34). These external annotations, as remarked in (22), are flags

192 useful to keep or discard a called variant. For instance, SNPiR implemented a series of

193 hard-filtering rules based on those annotations in a subsequent funneling process,

194 progressively reducing the number of potential false positive SNPs in their call set at the

195 cost of strongly reducing the overall number of called variants.

\section{Model validation}

197 After training the RF model, we tested its predictive performance against 3 other

198 alternative workflows on 10 skin fibroblasts samples from Neuromics, and on sample

199 NA12878. Specifically, we evaluated its predictive performance in terms of precision and

200 recall against the ground truth constituted by genomic high-quality variant calls. Those 
201 are the four considered alternatives, including SmartRNAseqCaller.

202

203 - GATK Best practices recommendations for calling RNA-Seq variants.

204 - GATK Best practices recommendations plus SmartRNASeqCaller to validate

205 whether the model refines the initial RNA-Seq called variants.

206 - SNPiR, which is able to provide reliable calls for SNPs without being limited to

207 somatic variant detection.

208 - SNPiR-like hard filtering. In this alternative we assess the potential of simple 209 filtering scheme using annotated features for the model. In this workflow we 210 discarded all variants with an annotation of RNA-Edit, homopolymeric region, 211 repmask region, or intron-exon junction. This should serve as a proxy to 212 understand the impact of following a more sophisticated RNA-Seq variant calling approximation. Importantly, this approximation sets the baseline of the performed

216 Moreover, we processed 365 samples from GTEx consortium evaluating the impact of 217 including SmartRNASeqCaller on top of GATK best practices workflow. We used the 218 Analysis Freeze WGS variant calls that have been used in GTEx for eQTL and Allele 219 Specific Expression analyses (35) as true reference set. We measured the performances 220 by precision and recall, analyzing the effect both on the bulk of samples and tissue-wise 221 in order to highlight potential biases due to SmartRNASeqCalled being trained on 222 fibroblast samples.

223 Following commonly accepted practices from genomic data analysis, we focused on 224 regions covered by at least 8 reads. We chose this threshold as it should allow reliable 225 identification for heterozygous genotypes with sufficient sensitivity (22). All samples have 226 been processed using the human reference genome hs37d5 (37). 


\section{Code availability and execution requirements}

228 SmartRNASeqcaller is available at https://github.com/inab/SmartRNASeqCaller. It can be

229 downloaded and executed as a shell script with specific parameters to change its default

230 behaviour, and/or using software containers e.g. dockers, inside a nextflow workflow (29).

231 We expect to guarantee full analysis reproducibility following recommendations around

232 Open Science, Open Data and Open Source. An average run of SmartRNASeqCaller

233 with Nextflow implementation takes 46 minutes, using less than 4 GB RAM with 4 CPUs

234 in parallel.

\section{Results}

236 Our first goal was to train a reliable model to classify true germline variants using RNA237 Seq. Then we validated using three different independent datasets against three 238 commonly used workflows. As demonstrated below, SmartRNASeqCaller would enable

239 the use of RNA-Seq variant calling in the clinic practice by reducing the burden of false 240 positive calls.

SmartRNASeqcaler obtains better precision/recall results than state-of-the-art workflows on fibroblast samples

243 We proceeded to measure the SmartRNASeqCaller performance on variants from 10 244 independent samples from the same Neuromics cohort used for training. We used 245 SmartRNASeqCaller as predictor for all variants considering called variants using WGS 246 as the gold standard. Following broadly adopted practices $(19,19,30)$, we evaluated 247 single nucleotide variants in regions with a minimum coverage of 8 or more RNA-Seq 248 reads to reduce the impact of wrong calls due to the effect of random noise on low249 coverage areas.

250 We report the precision/recall results for the all available samples (10 for training set and 
25110 for validation set) in Figure 2. In the case of SmartRNASeqCaller we reported

252 separately the performance for the training and validation data sets to assess the model

253 robustness and identify potential signs of overfitting.

254 First, the GATK Best practices workflow has an overall good performance in terms of 255 average precision $(82.9 \% \pm 3.9 \%)$ and recall $(78.7 \% \pm 1.4 \%)$. Second, the GATK 256 workflow has a better performance than SNPiR for the whole data set when considering 257 average precision and recall with F1 measure (GATK: 0.81 vs SNPiR: 0.66 From Table 258 2). Third, when comparing the performance on the training and validation samples for 259 SmartRNASeqCaller we can observe that the model is robust to overfitting. The average 260 performance on the training set, albeit better, is not drastically different when compared to 261 the validation samples. Focusing on differential changes with respect to the baseline 262 established by the GATK best practices workflow (Suppl fig. 1), the overall impact of 263 SmartRNASeqCaller brings significant improvements in precision (on average $+9 \%$ for 264 the validation set) with a modest tradeoff in recall (on average $-0.9 \%$ for the validation 265 set). This pattern is observed consistently among training and validation samples. Finally, 266 when compared to naïve hard-filtering strategies, we can appreciate that the average 267 precision is marginally improved but the average recall drastically drops, showing how 268 naïve approaches end-up doing more harm than good. These results support the idea of 269 integrating complex patterns derived from different sources, rather than limiting to simpler 270 intersection or union operations, using strategies based on machine learning techniques

\section{SmartRNASeqCaller improves precision on sample NA1278}

272 As a further evaluation step to study the model generalization and to exclude specific 273 biases from the considered samples, we tested SmartRNASeqCaller on the publicly 274 available sample NA12878 from the GIAB Consortium. On one hand, we processed raw 275 RNA-Seq reads through the GATK best practices variant calling workflow to have a 
276 baseline calls set. Building on this set we applied SmartRNASeq as an additional step to

277 the GATK Best practices called variants for comparison against it, against SNPiR, and

278 against a naïve hard-filtering strategy. We used GIAB calls from DNA sequencing as the

279 ground truth to evaluate the RNA-Seq variant calling results.

280 Similarly to the previous analysis, in Figure $2 \mathrm{~B}$ we reported the performance in terms of

281 precision/recall obtained for SmartRNASeqCaller and other alternative approaches.

282 Similar results to the previously analysed 20 samples were obtained confirming the 283 general usability of our model. Importantly, the baseline established by the GATK best 284 practices workflow yielded better results than SNPiR. This brings in the discussion the 285 impact of previous steps e.g. choice of the alignment strategy as well as the impact of the 286 continuous improvement of external annotation sources.

287 Similarly to comparison for the Neuromics samples, the application of 288 SmartRNASeqCaller to the baseline results allows to significantly improve precision (8\%) 289 with a moderate trade-off in recall $(\sim 2 \%)$ achieving the best overall results, while the 290 naïve hard-filtering strategy confirms to be the worst performing algorithm due to its 291 drastic effect on the final recall of variants. The baseline values of precision/recall for 292 NA12878 are worse than the average values with Neuromics samples as absolute 293 values. Nevertheless, the change brought by SmartRNASeqCaller is robust and in the 294 same direction, showing how the model behaves consistently across different initial 295 conditions.

296 SmartRNASeqCaller is robust to both tissue-of-origin differences, and alignment 297 algorithm

298 We then assessed SmartRNASeqCaller performance on a large independent cohort from 299365 GTEx (8) samples with matching WGS data. We chose tissue from 5 tissues that 300 represent most biopsies in clinical settings: Whole Blood, Skin Sun Exposed, Adipose 
301 Subcutaneous, Skeletal Muscle, and Fibroblasts. These tissues have diverse

302 transcriptome complexity and may be a closer representation of datasets used for clinical

303 applications.

304 GTEx v7 data have been aligned using TopHat v1.4, rather than STAR v3.5.1, which we 305 used to align the training set for SmartRNASeqCaller. Thanks to this, we could test how 306 robust SmartRNASeqCaller is to alternative upstream workflows, as aligners present 307 systematic differences between them. This is a particularly challenging dataset since 308 TopHat 1.4 has been shown to have many limitations and artifacts when compared to 309 recent aligners like STAR or Hisat2 $(12,31)$.

310 In Figure 3A, precision/recall results comparing the performance of the baseline 311 TopHat+GATK workflow and SmartRNASeqCaller applied as an additional step to the 312 baseline TopHat+GATK workflow are presented. The overall effect of strong precision 313 improvement with small sensitivity loss observed in Figure 2 is maintained on GTEx data.

314 Indeed, SmartRNASeqCaller improves precision on average by $20.9 \%$, a 6.25 fold 315 greater than the reduction in recall $(3.2 \%)$.

316 In Figure 3B, we present the precision values separated by tissue and workflow. The 317 median precision values for the TopHat+GATK workflow strongly depend on the tissue of 318 origin, ranging from $61.4 \%$ for Whole Blood, to $73.9 \%$ for Skeletal Muscle. After the 319 application of SmartRNASeqCaller, the precision levels range increase and are more 320 compact ranging from $85.6 \%$ in Whole Blood to $89.1 \%$ in Skeletal Muscle samples, 321 reducing dramatically $(\sim 50 \%)$ the differences between tissues. Similarly to Figure 3B, we 322 present in Figure 3C recall values for tissue of origin and workflow. SmartRNASeqCaller 323 effect is stable across tissues, reducing the sensitivity on average by $3.2 \%$ while keeping 324 the average recall between $85 \%-90 \%$ for all analyzed tissues. This is important because 325 we are able to capture much more true germline variants with higher precision that the 326 standard baseline. 
328 In general terms, SmartRNASeqCaller strongly improves the overall precision of RNA-

329 Seq variant calling with a small cost of sensitivity, even for data generated with different

330 aligners and collected from different tissues in the body demonstrating its general

331 applicability.

\section{Discussion}

333 In this work we developed SmartRNASeqCaller, a random forest model to reliably 334 discriminate true germline variants from the rest using RNA-Seq. SmartRNASeqCaller 335 combines intrinsic variant characteristics, with external annotation sources in a unique 336 model able to reduce the burden of false positive calls from RNA-Seq.

337 We trained our model using more than 600'000 variants from 10 high-quality samples 338 with matching WGS data from Neuromics Consortium. We then validated it against a 339 dataset of 10 independent samples from the same cohort, as well as on an independent 340 validation set composed by the broadly used sample NA12878 from the GIAB Consortium 341 (24), and by 365 samples from GTEx consortium (8). In all cases, applying 342 SmartRNASeqCaller significantly reduced the number of false positive calls almost

343 halving the number, without hindering recall e.g. average $0.9 \%$ loss in recall for the 344 validation samples from GIAB and Neuromics, and $3.2 \%$ on GTEx samples. 345 SmartRNASeqCaller allowed to achieve the best precision/recall performance when 346 compared against state-of-the-art workflows e.g. GATK best practices variant calling 347 workflow and SNPiR (16).

348 A whole set of technical challenges for the wide adoption of RNA-Seq as a source of data 349 for germline variant calling have been described in the literature i.e. split read mapping, 350 alternative splicing, RNA-Edit, RNA polymerase errors during transcription, and allele 351 specific expression $(12,15,16)$. Several tools have now been released to address these 
352 task-specific challenges. Examples are tools such as STAR and Histat2 $(17,18)$, which

353 aim to improve read alignment; or REDITools and DeepRed, which are tools to detect

354 RNA Editing events (19,20). Resources like REDIPortal and RADAR $(25,27)$ collect

355 regions with evidence of RNA-Edit activity along the human genome and are a valuable

356 resource to spot potential false positive calls.

357 However, few workflows have been developed to reliably call and filter germline

358 mutations from RNA-Seq. Those developed though rely on a set of hard-filtering rules

359 implying a trade-off between quality and quantity of selected variants. Some examples

360 are eSNV-detect (21), SNPiR (12), and Opossum (22). eSNV-detect (21) combines

361 multiple aligners to reduce aligner-specific errors prior to the variant calling itself. Once

362 this step is completed, eSNV-detect calls variants using SAMtools (32). However, this

363 practice introduces significant computational costs and questions their use in routinary

364 analysis. SNPiR (12) uses BWA-aln (23) to map spliced reads combined with GATK

365 UnifiedGenotyper (24) to generate an initial set of variant calls, which are then filtered

366 using external annotations about variant characteristics e.g. RNA-Edit site, homopolymer

367 region, repmask site. This filtering allows to improve precision at the cost of reduced

368 sensitivity. Opossum (22) employs a different strategy by preprocessing and filtering

369 RNA-Seq raw data to make it suitable for haplotype-based variant calling with Platypus

370 (25). This strategy renders remarkable results, albeit limited to the easily aligned portion

371 of the genome. Moreover, a priori exclusion of all sites prone to RNA-Edit, which include

372 many true germline variant e.g. $25 \%$ of RNA-Edit positions in RADAR and REDI-Portal

373 databases are located in exonic areas overlap with documented DNA mutations in

374 GnomAD dataset (26), may limit the use of Opossum into routine clinical practice.

375 Methods evaluation in most of these works is not standardized and is heavily dependent

376 on the annotations used to determine the scope of analysis e.g. gene definitions,

377 inclusion or exclusion of specific regions/SNP type, publicly available gold standard 
378 dataset, etc. There is therefore a need to joint efforts in the community to standardize

379 those efforts including the definition of relevant datasets and metrics.

380 The main driver to develop SmartRNASeqCaller was to obtain the highest reliability for 381 variants called from RNA-Seq experiments for its use in routine clinical practice. For this

382 we focused on improving the precision of the generated variant calls. We first chose to 383 integrate heterogeneous and non-redundant variants features to generate a rich and 384 complex description of each variant. Tools like SNPiR use a similar approach to apply 385 simple filters to exclude variants if characterized by unreliable features, which improved 386 precision compared to baseline. However, a simple filtering strategy is unable to properly 387 exploit the potential of a rich and complex multidimensional space. It can generate a 388 strong tradeoff between precision and sensitivity that can be detrimental for tasks such as 389 diagnosis. For that, we chose to train a Random Forest classifier on more than 600'000 390 variants from 10 samples. We chose Random Forests because it has been previously 391 applied in complex scenarios with many training samples, producing remarkable results in 392 terms of precision and robustness including DNA variant calling (21). We then evaluated 393 SmartRNASeqCaller following standard practices of processing independent samples 394 from different studies to ensure the general usability of this model across a wide variety of 395 samples from different tissues, and different upstream alternative workflows to generate 396 the initial calls sets.

397 Here we show that switching from a naïve hard-filtering schema to a more powerful, data398 driven solution enabled a qualitative and quantitative improvement in terms of 399 precision/recall. When compared to a SNPiR-like strategies of filtering all variants 400 annotated by some unreliable characteristic, the drastic reduction in recall does not 401 compensate for the improvement in terms of precision. This effect is mostly due to the 402 improvement and expansion of available annotations since the SNPiR publication, as well 403 as to the quality filtering already implemented in the baseline workflow that removes 
404 plenty of unreliable variants from RNA-Seq.

405 SmartRNASeqCaller builds on existing literature for variant calling using RNA-Seq,

406 improving overall performances and trustworthiness of the obtained results. Nevertheless,

407 as noted in $(16,24)$, its discovery potential is inherently limited by the nature of RNA-Seq

408 experimental set-ups: there is no hope to detect variants in areas of the genome that are

409 not expressed. Similarly, tissue-specific gene expression can limit the discovery of

410 phenotypic-causing variants as many experiment tend to use easily accessible tissues

411 rather than the affected one. Those accessible tissues might not express the genes of

412 interest for dissecting the genetic causes of the observed phenotype. However, recent

413 results showed that it is possible to obtain reliable mutation profile data of not easy-to-

414 reach tissues from other accessible tissues by generating suitable reprogrammed cells

415 (18). How RNA-Seq data is obtained can also directly affect the sensitivity of our method

416 as nonsense variants can be missed as a result of the nonsense-mediated decay

417 mechanisms (33).

418 Despite these factors limiting the scope of potential discoveries from RNA-Seq, they can

419 simultaneously be turned into a powerful filter against noise. Provided that the sequenced

420 tissue is relevant for the studied disease, RNA-Seq variants can limit the focus to those

421 genes that actually are being used by the affected cells, as well as inferring if there are

422 "missing genes" e.g. genes that are normally expressed in the tissue that are not present

423 in the experiment when considering reference datasets.

424 An additional factor contributing towards the divergence between RNA-Seq variants and

425 variants extracted from DNA is the existence of genes in which only one parental allele is

426 expressed (16,34). Previous work in this direction suggests that only $5 \%-10 \%$ of human

427 genes are subject to monoallelic gene expression (34), which could account for up to half

428 of the missing recall in our results. Strategies to improve the overall recall will require then

429 restructuring baseline variant calling workflows, specifically about the calling and filtering 
criteria

431 Although SmartRNASeqCaller allows to drastically reduce false positives from the 432 analyzed data, similarly to other tools e.g. SNPiR, and approximations, our model may 433 miss to filter variants due to systematic errors in the preceding workflows. Different 434 strategies have been proposed to overcome those systematic errors including merging 435 results from multiple samples to exclude novel recurrent rare variants (34). However, we 436 believe that with a much wider and diverse training dataset, the occurrence of systematic 437 errors can be strongly reduced. Moreover, our model can easily incorporate extra 438 features that may characterize systematic errors e.g. DNA sequence surrounding each 439 variant, in future developments.

440 A go-to RNA-Seq reliable variant calling workflow like SmartRNASeqCaller can help

441 filtering out genomic variants that may look promising from DNA data analysis but are 442 either not expressed in the tissue of interest, or removed by post-transcriptional 443 modifications, reducing the burden of false positive calls and enhancing the diagnosis 444 potential of these analyses.

445 Importantly, an additional benefit of reliable RNA-Seq variant calling would allow to detect 446 post-transcriptional RNA-specific variants that are not present at genomic level but could 447 have functional effects by themselves and/or jointly with nearby genomic variants. 448 Accurate variant calling results can help investigating if RNA-Edit, generally not 449 considered as source of disease, may act detrimentally towards the cell. It is theoretically 450 possible to detect RNA-Edit events acting like germline variants for further annotation and 451 interpretation for disease generation (35).

453 Conclusions

454 Despite the limitations of calling genomic variants from RNA-Seq, our work demonstrates 455 improvements in the field of RNA-Seq variant calling to detect germline variants with high 
456 precision and recall using appropriate machine learning tools.

457 SmartRNASeqCaller can be a go-to tool for reliable variant calling from RNA-Seq, with

458 the potential to enhance diagnostic yield and have better disease characterization in the

459 tissue of interest. SmartRNASeqCaller allows to harness information from RNA-Seq and

460 to generate a very precise calls set with good sensitivity. These characteristics are of

461 paramount importance in clinical settings and can provide relevant benefits. RNA-Seq

462 can be used to integrate DNA mutation information with tissue specific results providing

463 an independent source of information to filter and validate disease-causing candidate

464 variants.

465 Furthermore it can palliate the absence of genomic data for specific samples, presenting

466 a viable way to extract a reliable variant calls, and generate a new knowledge base of

467 RNA mutations. This could allow RNA-Seq samples processing for tissues cohorts in

468 clinic to extract a very precise and context-specific mutational landscape without requiring

469 additional DNA sequencing.

470 Finally, SmartRNASeqCaller can be used as an additional step of any existing variant

471 calling workflow. This makes possible to even reanalyze existing cohorts with the goal of

472 detecting germline variations without requiring expensive computation.

\section{Declarations}

\section{Ethics approval and consent to participate}

475 Not applicable. All samples processed in this work come from consortia in which the

476 consent has been explicitly granted for research purposes.

\section{Consent for publication}

$478 \quad$ Not applicable 
479

480

481

482

483

484

485

486

487

488

\section{Availability of data and material}

Project name: SmartRNASeqCaller

Project home page: https://github.com/inab/SmartRNASegCaller

$$
\text { Operating system(s): Platform independent }
$$

Programming language: Python, Bash, Nextflow, R

Other requirements: GATK 3.6-0, Samtools, Bcftools, Bedtools,tabix,Python 2.7: (pysam, pandas), R 3.5 .0 (caret, ranger). Optional: Docker

$$
\text { License: GNU GPLv3 }
$$

\section{Datasets availability:}

- GIAB NA12878 data are available at : https://jimb.stanford.edu/giab-resources

- Neuromics cohort: The data that support the findings of this study are available from Neuromics consortium but restrictions apply to the availability of these data, which were used under license for the current study, and so are not publicly available. Data are however available from the authors upon reasonable request and with permission of Neuromics consortium. https://rd-neuromics.eu/projectwelcome/

- GTEx data: The data that support the findings of this study are available from GTEx Consortium but restrictions apply to the availability of these data, which were used under license for the current study, and so are not publicly available. Data are however available from the authors upon reasonable request and with permission of GTEx consortium. https://gtexportal.org/home/datasets

\section{List of abbreviations:}

RF: Random Forest

WES: Whole Exome Sequencing 
504 WGS: Whole Genome Sequencing

505 GATK: Genome Analysis ToolKit

506 GIAB : Genome In a Bottle

507 GTEx : Genotype Tissue Expression

508 Competing interests

509 The authors declare that they have no competing interests

510 Funding

511 RD-Connect has been established thanks to the funding from the European Community's

512 Seventh Framework Program (FP7) under grant agreement number 305444 "RD-

513 CONNECT: An integrated platform connecting registries, biobanks and clinical

514 bioinformatics for rare disease research. The Central Node at the Barcelona

515 Supercomputing Center (BSC) is a member of the Spanish National Bioinformatics

516 Institute (INB), ISCIII-Bioinformatics platform and is supported by grant PT17/0009/0001,

517 of the Acción Estratégica en Salud 2013-2016 of the Programa Estatal de Investigación

518 Orientada a los Retos de la Sociedad, funded by the Instituto de Salud Carlos III (ISCIII)

519 and European Regional Development Fund (ERDF). This work was funded by ELIXIR,

520 the research infrastructure for life-science data.

\section{Authors' contributions}

522 M.B: Designed the algorithm, performed training and validation of the data, write the first

523 version of the manuscript. A.V: Designed the algorithm and validation strategy, S.C.G

524 Designed the algorithm and validation strategy, write the final version of the manuscript.

525 All authors read and approved the final manuscript. 


\section{Acknowledgements}

527 We are grateful to Ana Topf and Steve Laurie for facilitating the access to the data from

528 the Neuromics project. We also thank insightful comments over preliminary versions of

529 this work to Marta Melé and Jennifer Harrow.

\section{FIGURES}

531 Figure 1: Random forest model construction and iterative feature selection.

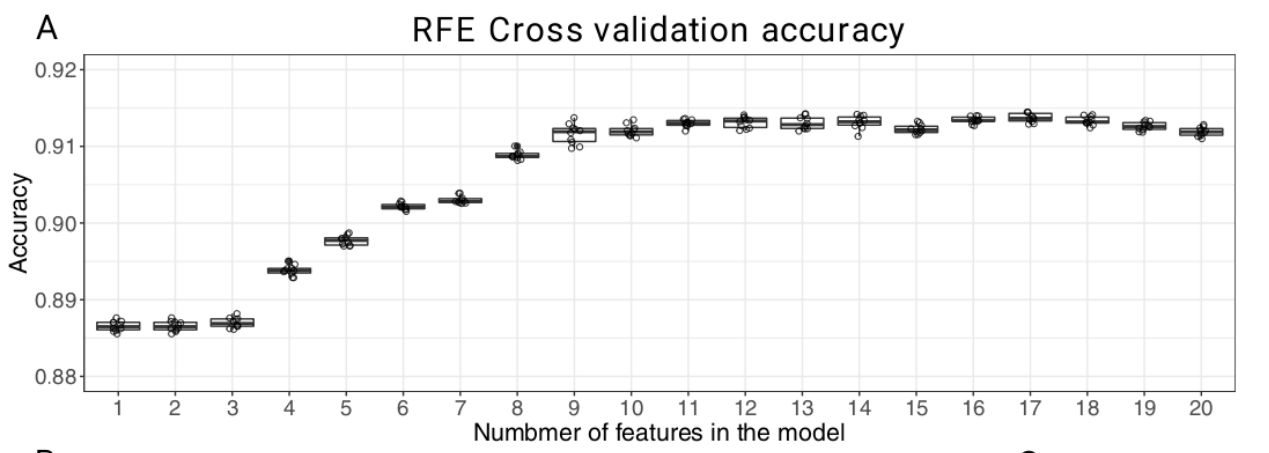

B tSNE Representation of 20 features

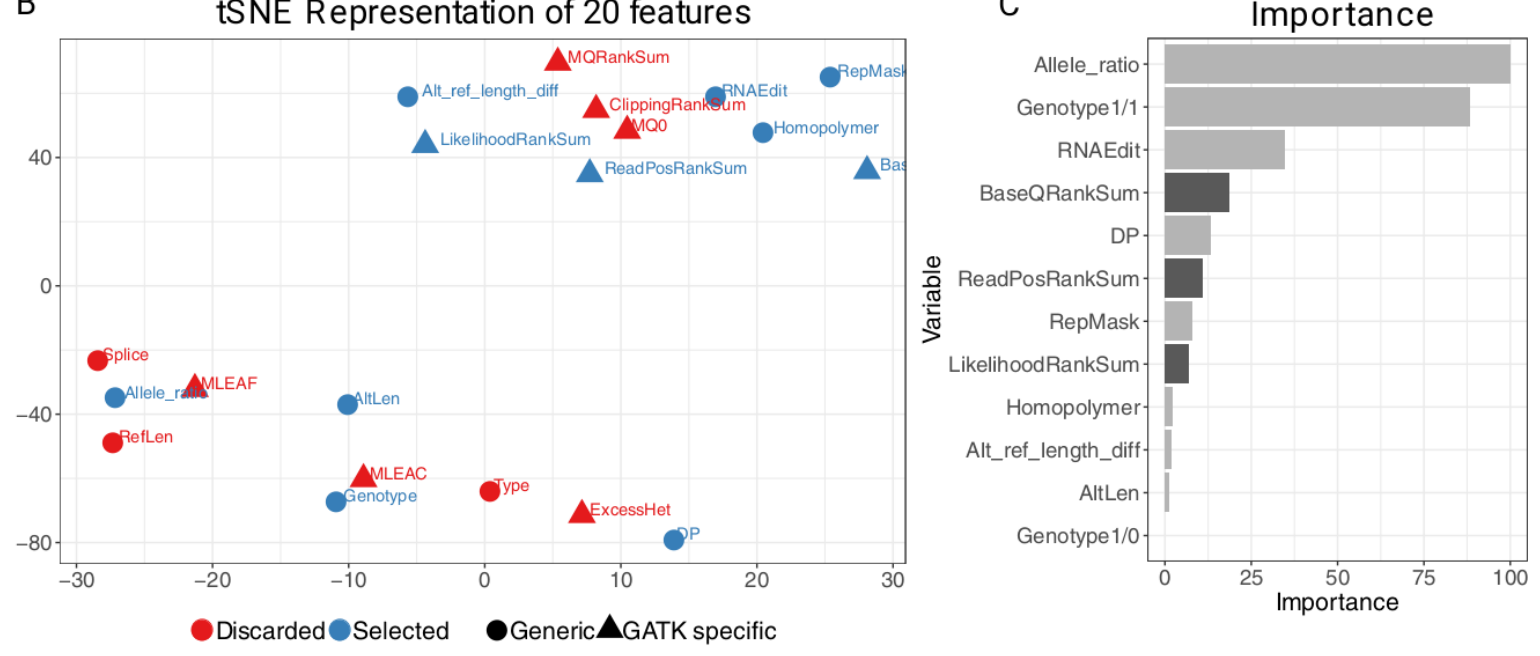

532

533 A) Training performances for recursive feature elimination process. From 11 features on

534 there is no apparent benefit in terms of classification accuracy.

535 B) tSNE representation of the 20 features studied using the training data set. Features

536 are color and shape coded to reflect if they are part of the final model, and if they are

537 generic e.g. intrinsic and contextual properties, or GATK specific. All excluded features 
538 are very close to at least one selected one, suggesting that their information content was

539 redundant.

540 C) Variant importance for the prediction model. Light gray darks represent generic

541 annotations, Darker grey bars represent GATK specific annotations.

542

543 Figure 2: Precision/Recall results on Neuromics and NA12878 GIAB samples
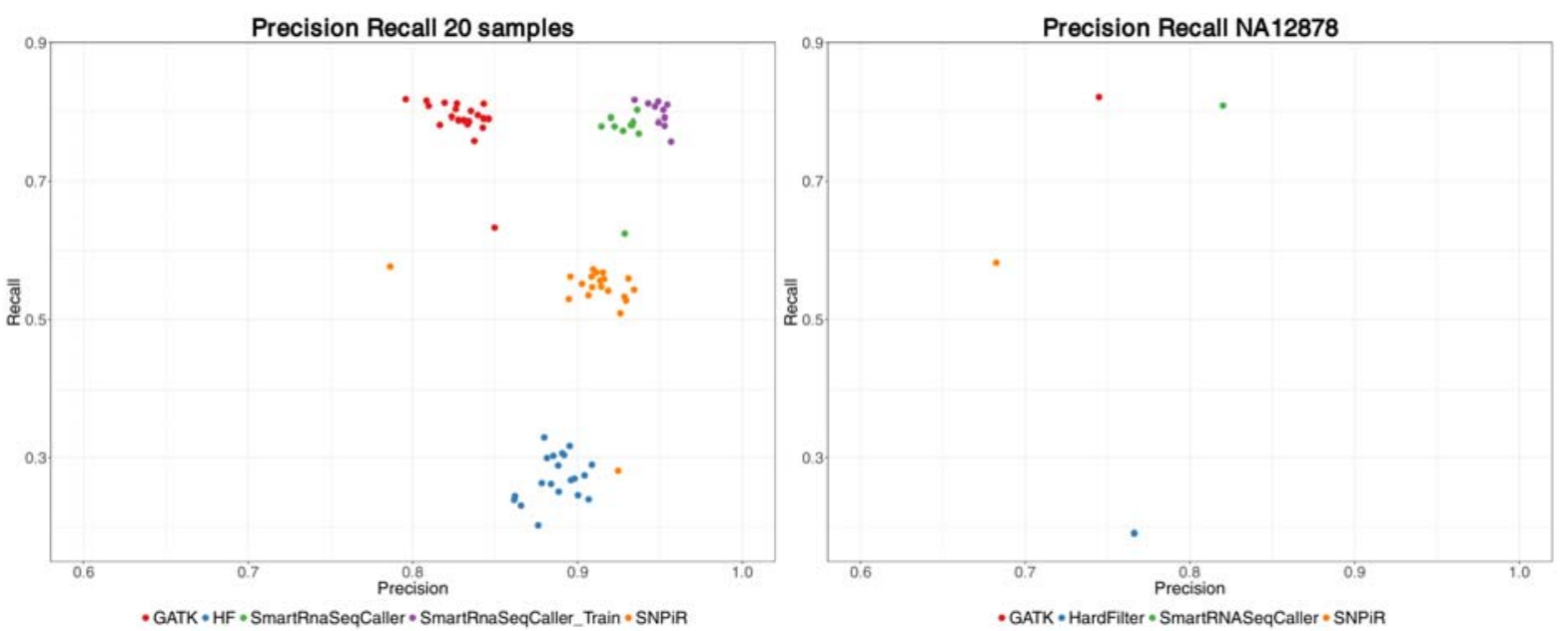

545 A) Precision/recall results analysing two separated sets of 10 samples each from the

546 same cohort from the Neuromics consortium, which are available at the RD-Connect

547 platform. It compares the SmartRNASeqCaller application against the baseline GATK

548 Best practices variant calling workflow, against an alternative naive filtering method

549 depicted as Hard Filtering, and against SNPiR. We report the results for the training and

550 validation samples for SmartRNASeqCaller separately to show that there is not sign of

551 overfitting to the model. Moreover, we can observe how the strong improvement of

552 precision at a moderate loss of recall behavior is conserved for the validation set of

553 samples, which have not been used at all for generating the Random Forest model As

554 expected, the precision/recall values for the samples in the training set are better than for

555 the validation samples, but the overall effect is similar and robust on the 10 validation

556 samples. Indeed, for the training data set SmartRNASeqCaller achieves $+12.0 \%$ 
557 precision, and $0.1 \%$ less recall while that for the validation data set it obtains a $+9.3 \%$

558 precision and $0.9 \%$ less recall compared to the GATK best practices workflow

559 (supplementary figure 1).

560 B) Precision/recall results after analysing sample NA12878. It compares

561 SmartRNASeqCaller against the baseline GATK best practices variant calling workflow,

562 against an alternative naive filtering method depicted as Hard Filtering, and against

563 "SNPiR". We can observe how the strong improvement of precision at a moderate loss of

564 recall behavior is conserved in this independent sample as well. Here too, the overall

565 relationships among method are conserved, confirming the results previously obtained on

566 the 20 samples from the Neuromics Consortium.

567 Figure 3: Precision/Recall on 365 GTEx samples 


\section{A}

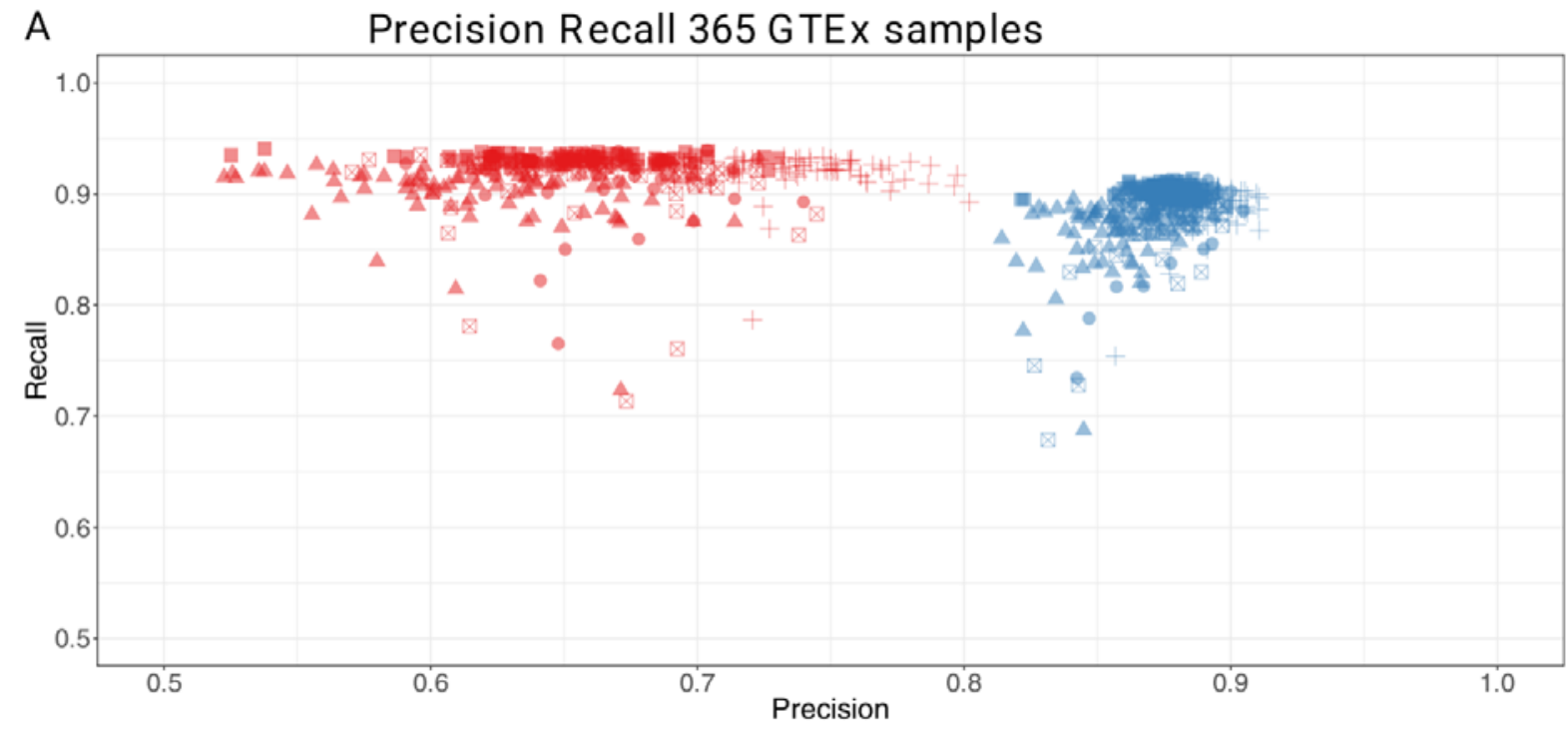

- Adipose Tissue $\triangle$ Blood $₫$ Fibroblasts + Muscle $\otimes$ Skin

B

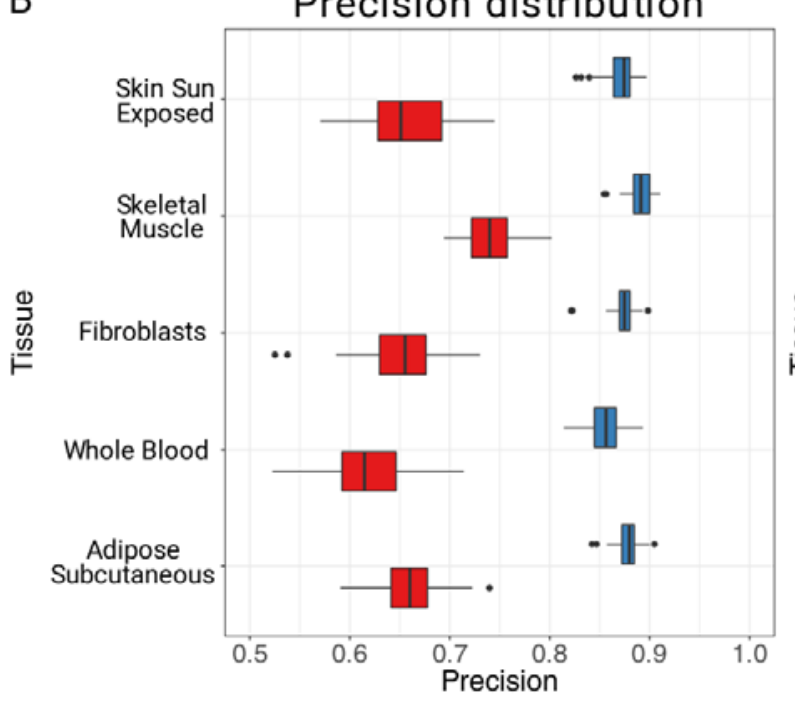

TopHat + GATK @SmartRNASeqCaller
- TopHat + GATK •SmartRNASeqCaller

\section{$\mathrm{C}$}

Recall distribution

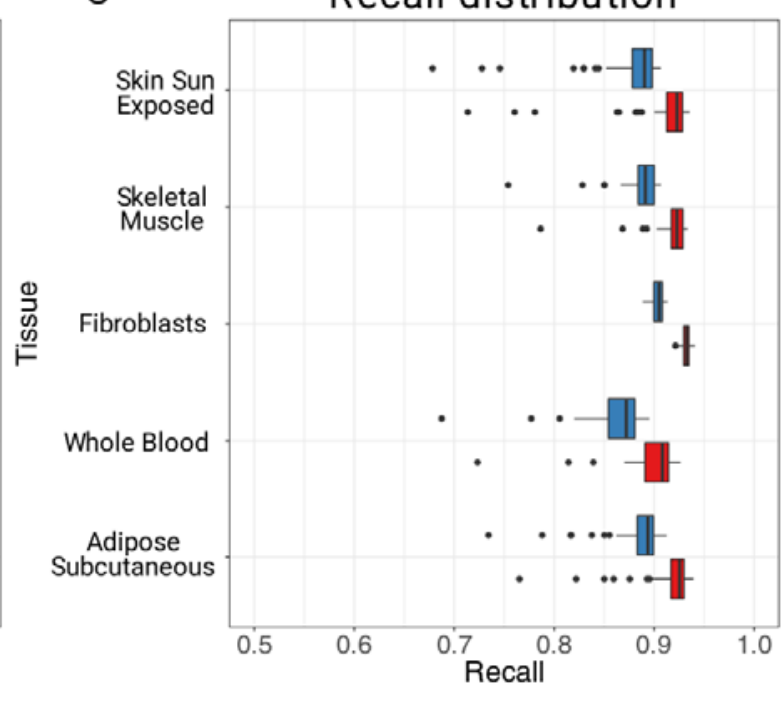

@TopHat + GATK @SmartRNASeqCaller

568

569 A) Precision/recall results analysing 365 tissue samples from GTEx cohort. Samples are

570 from five different tissues and 73 patients. Precision/recall plot comparing TopHat+GATK

571 Best practices variant calling workflow against SmartRNASeqCaller applied on

572 TopHat+GATK results. SmartRNASeqCaller shows a strong effect improving precision on

573 average by $20.9 \%$, reducing Recall by $3.2 \%$ on average.

574 B) Boxplots comparing precision values for GATK best practices and

575 SmarRNASeqCaller. We observe how samples from different tissues have different

576 burden of false positives in GATK. After SmartRNASeqCaller application the differences 
577 are less evident and the boxplots overlap across tissues. C) Boxplots comparing Recall

578 values for GATK best practices and SmarRNASeqCaller. On average, the application of

579 SmartRNASeqCaller reduces recall by $3.2 \%$. The impact of SmartRNASeqCaller is to

580 increase the overall precision, levelling the performance across tissues close to $90 \%$,

581 simultaneously keeping high levels of Recall (between 85 and 90\%).

\section{Tables}

583 Table 1. Features considered to train the random forest model.

\begin{tabular}{|c|c|c|c|}
\hline & Name & Selected? & Extra information \\
\hline \multirow{7}{*}{$\begin{array}{l}\text { Intrinsic } \\
\text { properties }\end{array}$} & Allele ratio & Yes & Alternative allele percentage \\
\hline & Alt Len & Yes & Length of the alternative allele \\
\hline & Genotype & Yes & Heterozygous or homozygous call \\
\hline & DP & Yes & Depth of coverage \\
\hline & Ref-Alt Len & Yes & $\begin{array}{l}\text { Length difference of alternative and } \\
\text { reference alleles }\end{array}$ \\
\hline & RefLen & No & Length of reference allele \\
\hline & Type & No & SNP / Indel \\
\hline \multirow{2}{*}{$\begin{array}{l}\text { Contextual } \\
\text { features }\end{array}$} & RNA-Edit & Yes & $\begin{array}{l}\text { Annotated as RNA-Edit event in } \\
\text { databases }\end{array}$ \\
\hline & RepMask & Yes & $\begin{array}{l}\text { Included in RepeatMasker track from } \\
\text { UCSC Genome Browser }\end{array}$ \\
\hline
\end{tabular}




\begin{tabular}{|c|c|c|c|}
\hline & Homopolymer & Yes & $\begin{array}{l}\text { Is the variant within a homopolymeric } \\
\text { region of genome ( } 5 \text { bases or more) }\end{array}$ \\
\hline & SpliceSite & No & $\begin{array}{l}\text { Is the variant within } 4 \text { nucleotide } \\
\text { distances from an exon-intron } \\
\text { junction }\end{array}$ \\
\hline \multirow{5}{*}{$\begin{array}{l}\text { GATK-specific } \\
\text { Annotations }\end{array}$} & BaseQRankSum & Yes & $\begin{array}{l}\text { Compares the base qualities of the } \\
\text { data supporting the reference allele } \\
\text { with those supporting any alternate } \\
\text { allele. }\end{array}$ \\
\hline & ReadPosRankSum & Yes & $\begin{array}{l}\text { Tests whether there is evidence of } \\
\text { bias in the position of alleles within } \\
\text { the reads that support them, between } \\
\text { the reference and alternate alleles. }\end{array}$ \\
\hline & LikelihoodRankSum & Yes & $\begin{array}{l}\text { Compares the likelihood of reads to } \\
\text { their best haplotype match, between } \\
\text { reads that support the reference } \\
\text { allele and those that support the } \\
\text { alternate allele. }\end{array}$ \\
\hline & ClippingRankSum & No & $\begin{array}{l}\text { Tests whether the data supporting } \\
\text { the reference allele shows more or } \\
\text { less base clipping (hard clips) than } \\
\text { those supporting the alternate allele. }\end{array}$ \\
\hline & ExcessHet & No & Estimates excess heterozygosity in a \\
\hline
\end{tabular}




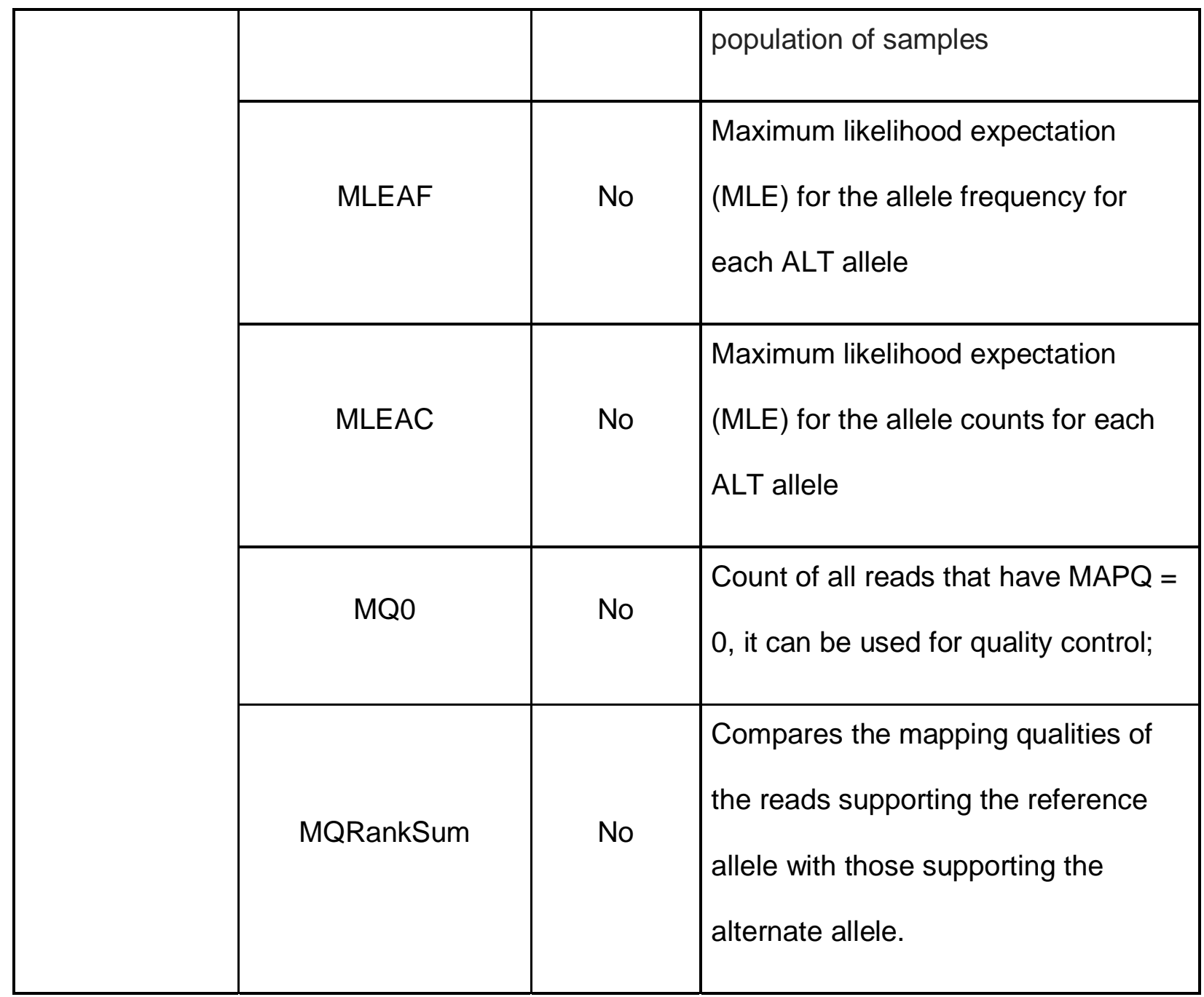

584 This table contains a brief description of all features, as well as if they have been selected

585 for the final SmartRNASeqCaller model. Features are split by type, and the selected ones

586 are sorted by the relevant importance for the prediction model, as from Figure $1 \mathrm{C}$.

589 Table 2: Summary of F1 statistic on Neuromics samples

\begin{tabular}{|c|c|c|c|c|c|}
\hline F1 & Hard & GATK & $\begin{array}{c}\text { SmartRNASeqCaller } \\
\text { Train }\end{array}$ & $\begin{array}{c}\text { SmartRNASeqCaller } \\
\text { Test }\end{array}$ & SNPiR \\
\hline Mean & 0.41 & 0.81 & 0.87 & 0.84 & 0.66 \\
\hline
\end{tabular}




\begin{tabular}{|c|c|c|c|c|c|}
\hline Median & 0.41 & 0.81 & 0.87 & 0.85 & 0.68 \\
\hline $\begin{array}{l}\text { Standard } \\
\text { Deviation }\end{array}$ & 0.04 & 0.02 & 0.01 & 0.03 & 0.08 \\
\hline Minimum & 0.33 & 0.73 & 0.85 & 0.75 & 0.43 \\
\hline Maximum & 0.48 & 0.83 & 0.88 & 0.86 & 0.70 \\
\hline
\end{tabular}

590 F1 measure(geometric mean of precision and recall on 20 samples from a cohort from

591 the Neuromics Consortium. From this summary statistic we can infer how the baseline

592 GATK variant calling workflow achieves better results than the simple hard filtering

593 strategy, and the SNPiR algorithm as well. The application of SmartRNASeqCaller to the

594 GATK best practices workflow, allows to further improve the F1 results. We split train and

595 validation values for SmartRNASeqCaller, to avoid bias of the training samples on the

596 overall result. 


\section{References}

598 1. Clark MM, Stark Z, Farnaes L, Tan TY, White SM, Dimmock D, et al. Meta-analysis

599 of the diagnostic and clinical utility of genome and exome sequencing and

600 chromosomal microarray in children with suspected genetic diseases. Npj Genomic

$601 \quad$ Med. 2018 Jul 9;3(1):16.

602 2. Nguyen MT, Charlebois K. The clinical utility of whole-exome sequencing in the

603 context of rare diseases - the changing tides of medical practice. Clin Genet. 2015

604 Oct;88(4):313-9.

605 3. Rau A, Flister M, Rui H, Auer PL. Exploring drivers of gene expression in the Cancer 606 Genome Atlas. Bioinformatics. 2019 Jan 1;35(1):62-8.

607 4. Thorsson V, Gibbs DL, Brown SD, Wolf D, Bortone DS, Ou Yang T-H, et al. The 608 Immune Landscape of Cancer. Immunity. 2018 17;48(4):812-830.e14.

609 5. Bailey MH, Tokheim C, Porta-Pardo E, Sengupta S, Bertrand D, Weerasinghe A, et 610 al. Comprehensive Characterization of Cancer Driver Genes and Mutations. Cell. 6112018 Apr 5;173(2):371-385.e18.

612 6. The 1000 Genomes Project Consortium. A global reference for human genetic 613 variation. Nature. 2015 Oct;526(7571):68-74.

$6147 . \quad$ The UK10K project identifies rare variants in health and disease. Nature. 2015 Oct $615 \quad 1 ; 526(7571): 82-90$.

616 8. The Genotype-Tissue Expression (GTEx) project | Nature Genetics [Internet]. [cited 6172018 Dec 3]. Available from: https://www.nature.com/articles/ng.2653

618 9. Zawati MH, Parry D, Thorogood A, Nguyen MT, Boycott KM, Rosenblatt D, et al.

619 Reporting results from whole-genome and whole-exome sequencing in clinical 620 practice: a proposal for Canada? J Med Genet. 2014 Jan;51(1):68-70.

621 10. Bosio M, Drechsel O, Rahman R, Muyas F, Rabionet R, Bezdan D, et al. eDiVA622 Classification and prioritization of pathogenic variants for clinical diagnostics. Hum 
Mutat [Internet]. 2019 [cited 2019 May 29];0(0). Available from:

624 https://onlinelibrary.wiley.com/doi/abs/10.1002/humu.23772

625 11. Byron SA, Van Keuren-Jensen KR, Engelthaler DM, Carpten JD, Craig DW.

626 Translating RNA sequencing into clinical diagnostics: opportunities and challenges.

$627 \quad$ Nat Rev Genet. 2016 May;17(5):257-71.

628 12. Sahraeian SME, Mohiyuddin M, Sebra R, Tilgner H, Afshar PT, Au KF, et al. Gaining 629 comprehensive biological insight into the transcriptome by performing a broad-

630 spectrum RNA-seq analysis. Nat Commun. 2017 05;8(1):59.

631 13. Xu C. A review of somatic single nucleotide variant calling algorithms for next-

632 generation sequencing data. Comput Struct Biotechnol J. 2018 Jan 1;16:15-24.

633 14. Conesa A, Madrigal P, Tarazona S, Gomez-Cabrero D, Cervera A, McPherson A, et

634 al. A survey of best practices for RNA-seq data analysis. Genome Biol [Internet].

6352016 [cited 2018 Dec 3];17. Available from:

636 https://www.ncbi.nlm.nih.gov/pmc/articles/PMC4728800/

637 15. Han Y, Gao S, Muegge K, Zhang W, Zhou B. Advanced Applications of RNA

638 Sequencing and Challenges. Bioinforma Biol Insights. 2015 Nov 15;9(Suppl 1):29_

$639 \quad 46$.

640 16. Piskol R, Ramaswami G, Li JB. Reliable Identification of Genomic Variants from

641 RNA-Seq Data. Am J Hum Genet. 2013 Oct 3;93(4):641-51.

642 17. Cummings BB, Marshall JL, Tukiainen T, Lek M, Donkervoort S, Foley AR, et al.

643 Improving genetic diagnosis in Mendelian disease with transcriptome sequencing.

$644 \quad$ Sci Transl Med. 2017 19;9(386).

645 18. Gonorazky HD, Naumenko S, Ramani AK, Nelakuditi V, Mashouri P, Wang P, et al.

646 Expanding the Boundaries of RNA Sequencing as a Diagnostic Tool for Rare

647 Mendelian Disease. Am J Hum Genet. 2019 Mar 7;104(3):466-83.

648 19. Oikkonen L, Lise S. Making the most of RNA-seq: Pre-processing sequencing data 
with Opossum for reliable SNP variant detection. Wellcome Open Res [Internet]. 2017 Mar 17 [cited 2018 Dec 3];2. Available from: https://www.ncbi.nlm.nih.gov/pmc/articles/PMC5322827/

20. Tang X, Baheti S, Shameer K, Thompson KJ, Wills Q, Niu N, et al. The eSNV-detect: a computational system to identify expressed single nucleotide variants from transcriptome sequencing data. Nucleic Acids Res. 2014 Dec 16;42(22):e172.

21. Ho DSW, Schierding W, Wake M, Saffery R, O'Sullivan J. Machine Learning SNP Based Prediction for Precision Medicine. Front Genet [Internet]. 2019 [cited 2019

22. Triantafyllidis AK, Tsanas A. Applications of Machine Learning in Real-Life Digital Health Interventions: Review of the Literature. J Med Internet Res. 2019;21(4):e12286.

23. Lochmüller H, Badowska DM, Thompson R, Knoers NV, Aartsma-Rus A, Gut I, et al. RD-Connect, NeurOmics and EURenOmics: collaborative European initiative for rare

24. Zook JM, Chapman B, Wang J, Mittelman D, Hofmann O, Hide W, et al. Integrating human sequence data sets provides a resource of benchmark SNP and indel genotype calls. Nat Biotechnol. 2014 Mar;32(3):246-51.

25. Picardi E, D’Erchia AM, Lo Giudice C, Pesole G. REDlportal: a comprehensive

26. Karolchik D, Hinrichs AS, Furey TS, Roskin KM, Sugnet CW, Haussler D, et al. The

672 UCSC Table Browser data retrieval tool. Nucleic Acids Res. 2004 Jan 1;32(Database issue):D493-496.

674 27. Ramaswami G, Li JB. RADAR: a rigorously annotated database of A-to-I RNA 
675 editing. Nucleic Acids Res. 2014 Jan 1;42(D1):D109-13.

676 28. Melé M, Ferreira PG, Reverter F, DeLuca DS, Monlong J, Sammeth M, et al. The

677 human transcriptome across tissues and individuals. Science. 2015 May

$678 \quad 8 ; 348(6235): 660-5$.

679 29. Di Tommaso P, Chatzou M, Floden EW, Barja PP, Palumbo E, Notredame C.

680 Nextflow enables reproducible computational workflows. Nat Biotechnol. 2017 Apr

$681 \quad 11 ; 35: 316-9$.

682 30. Laurie S, Fernandez $\square$ Callejo M, Marco $\square$ Sola S, Trotta J, Camps J, Chacón A, et al.

683 From Wet $\square$ Lab to Variations: Concordance and Speed of Bioinformatics Pipelines

684 for Whole Genome and Whole Exome Sequencing. Hum Mutat. 2016

685 Dec;37(12):1263-71.

686 31. Baruzzo G, Hayer KE, Kim EJ, Di Camillo B, FitzGerald GA, Grant GR. Simulationbased comprehensive benchmarking of RNA-seq aligners. Nat Methods. 2017

$688 \quad$ Feb;14(2):135-9.

689 32. Li H, Durbin R. Fast and accurate short read alignment with Burrows-Wheeler 690 transform. Bioinformatics. 2009 Jul 15;25(14):1754-60.

691 33. Behm-Ansmant I, Kashima I, Rehwinkel J, Saulière J, Wittkopp N, Izaurralde E. 692 mRNA quality control: an ancient machinery recognizes and degrades mRNAs with 693 nonsense codons. FEBS Lett. 2007 Jun 19;581(15):2845-53.

694 34. Chess A. Mechanisms and consequences of widespread random monoallelic 695 expression. Nat Rev Genet. 2012 May 15;13(6):421-8.

696 35. Meier JC, Kankowski S, Krestel H, Hetsch F. RNA Editing-Systemic Relevance and 697 Clue to Disease Mechanisms? Front Mol Neurosci [Internet]. 2016 Nov 23 [cited 6982019 Apr 10];9. Available from:

699 https://www.ncbi.nlm.nih.gov/pmc/articles/PMC5120146/ 